\title{
Caracterização das Práticas Integrativas e Complementares na Atenção Primária a Saúde no Estado do Pará
}

\author{
Characterization of Integrative and Complementary Practices in Primary Health Care in \\ State of Para
}
Caracterización de las Prácticas Integradoras y Complementarias en la Atención Primaria de Salud en el Estado de Pará

Maria Pantoja Moreira ${ }^{1}$, Marcos Valério Santos da Silva ${ }^{1}$, Luann Wendel Pereira de Sena ${ }^{1 *}$.

\section{RESUMO}

Objetivo: Caracterizar as ofertas das Práticas Integrativas e Complementares (PIC) na Atenção Primária a Saúde no Estado do Pará. Métodos: Trata-se de um estudo transversal, avaliativo, com abordagem quantitativa, do tipo normativo, a partir do banco de dados de base nacional da etapa de avaliação externa do Programa de Melhoria do Acesso e da Qualidade da Atenção Básica (PMAQ-AB). Resultados: Os resultados demonstraram um total de 125 munícipios (86,8\%) que aderiram ao PMAQ-AB. O Ciclo 1, não apresentou registros das PIC e os dados sugiram a partir do Ciclo 2. O Ciclo 3, demonstrou uma maior participação dessas práticas $(59,72 \%)$. Os municípios paraenses apresentaram uma boa estrutura física e realizaram educação permanente com os profissionais da saúde. A medicina tradicional chinesa/acupuntura foi a PIC que mais prevaleceu (7,69\%). Após a inserção de novas práticas, houve um aumento significativo do uso (116,2\%) e o seu desenvolvimento, ocorreu por processos diversos, tais como: educação permanente, consultas individuais e reuniões de grupos terapêuticos. Conclusão: $O$ Estado do Pará apresentou uma crescente na oferta das PIC na Atenção Primária a Saúde e a publicação da Portaria do MS nำ 849/2017, favoreceu o aumento nos municípios estudados.

Palavras-chave: Práticas Integrativas e Complementares, Atenção Primária a Saúde, Estado do Pará.

\begin{abstract}
Objective: To characterize the offers of Integrative and Complementary Practices (PIC) in Primary Health Care in the State of Para. Methods: This is a cross-sectional, evaluative study, with a quantitative approach, of the normative type, from the database of national basis of the external evaluation stage of the Program for the Improvement of Access and Quality of Primary Care (PMAQ-AB). Results: The results showed a total of 125 municipalities (86.8\%) who joined the PMAQ-AB. Cycle 1 did not present any PIC records and the data suggested from Cycle 2. Cycle 3 demonstrated a greater participation of these practices $(59.72 \%)$. The municipalities of Para had a good physical structure and carried out permanent education with health professionals. Traditional Chinese medicine/acupuncture was the most prevalent ICP (7.69\%). After the insertion of new practices, there was a significant increase in use (116.2\%) and its development occurred through different processes, such as: permanent education, individual consultations and meetings of therapeutic groups. Conclusion: The State of Para showed an increase in the supply of PICs in Primary Health Care and the publication of Ordinance of MS $n \circ 849 / 2017$, favored the increase in the municipalities studied.
\end{abstract}

Key words: Integrative and Complementary Practices, Primary Health Care, State of Pará.

\section{RESUMEN}

Objetivo: Caracterizar la oferta de Prácticas Integrativas y Complementarias (PIC) en Atención Primaria de Salud en el Estado de Pará. Métodos: Se trata de un estudio evaluativo, transversal, con enfoque cuantitativo, de tipo normativo, a partir de la base de datos de base nacional de la etapa de evaluación externa del

1 Universidade Federal do Pará, Belém - PA. *E-mail: luannsena@gmail.com

SUBMETIDO EM: 2/2021

ACEITO EM: 3/2021

PUBLICADO EM: $3 / 2021$ 
Programa de Mejoramiento del Acceso y Calidad de la Atención Primaria (PMAQ-AB). Resultados: Los resultados arrojaron un total de 125 municipios $(86,8 \%)$ que se incorporaron al PMAQ-AB. El ciclo 1 no presentó registros PIC y los datos sugeridos del ciclo 2 . El ciclo 3 demostró una mayor participación de estas prácticas $(59,72 \%)$. Los municipios de Pará tenían una buena estructura física y realizaban educación permanente con profesionales de la salud. La medicina tradicional china/acupuntura fue la PIC más prevalente $(7,69 \%)$. Luego de la inserción de nuevas prácticas, hubo un incremento significativo en el uso $(116,2 \%)$ y su desarrollo se dio a través de diferentes procesos, tales como: educación permanente, consultas individuales y reuniones de grupos terapéuticos. Conclusión: El Estado de Pará presentó un incremento en la oferta de PIC en Atención Primaria de Salud y la publicación de la Ordenanza de MS no 849/2017, favoreció el incremento en los municipios estudiados.

Palabras clave: Prácticas Integrativas y Complementarias, Atención Primaria de Salud, Estado de Pará.

\section{INTRODUÇÃO}

No decorrer do tempo, as práticas em saúde basearam-se em um modelo biomédico caracterizado pela centralização na doença, passando a tratar o corpo em partes cada vez menores, reduzindo a saúde a um processo mecânico, desvalorizando o paciente e uniformizando o cuidado através de intervenções clínicas severas e utilização contínua de medicamentos. Esse protótipo de atenção à saúde, dito como tradicional, passou a ser questionado, devido se afastar de um modelo baseado na singularidade do indivíduo. Com base nisso, os cuidados clínicos voltados a atenção ao paciente começaram a ser valorizados, pois, apresenta-se a humanização da medicina e a integralidade no cuidado por englobar dimensões biológicas, psicológicas, socioculturais, econômicas, ambientais e políticas (TESSER CD, et al., 2018).

As Práticas Integrativas e Complementares (PIC) buscam impulsionar os mecanismos naturais de prevenção de doenças e restabelecimento da saúde por meio de tecnologias eficazes e seguras, com ênfase na escuta acolhedora, no desenvolvimento do vínculo terapêutico e na integração do ser humano com o meio ambiente e a sociedade (BRASIL, 2018).

Por conta disso, a Organização Mundial da Saúde (OMS), vem incentivando o uso das PIC por seus países membros, através da criação de um documento normativo em prol do uso racional, moderado e integrado nas terapias não ortodoxas no sistema nacional de atenção à saúde (BRASIL, 2017).

Ao longo dos anos, as PIC vêm ganhando notoriedade devido os seus resultados benéficos. Há nível mundial, a expansão das PIC ocorreu devido os benefícios que essas práticas detêm, bem como a valorização do indivíduo como centro do cuidado, a busca por meios terapêuticos simples, menos dependentes de tecnologia, baixo valor econômico, eficácia maior ou igual nas situações comuns de adoecimento e a busca pela autonomia do usuário, com foco central na saúde e não na doença (SOUSA LA e BARROS NF, 2018).

No Brasil, $56 \%$ dos municípios já fazem o uso das PIC e o seu processo de institucionalização no Sistema Único de Saúde (SUS) ocorreu em 2017-2018, fazendo com que, 80\% de sua utilização ocorra na Atenção Primária a Saúde (APS) (TESSER CD, et al., 2018). Mesmo com todos esses reconhecimentos, a Política Nacional de Práticas Integrativas e Complementares (PNPIC), ainda demonstra-se ser ainda uma política frágil e com pouca visibilidade, quando comparadas a outras políticas públicas (SOUZA IN, et al., 2020).

A PNPIC foi criada em 2006 e instituiu no SUS abordagens de cuidado integral à população por meio de recursos terapêuticos, entre eles fitoterapia, acupuntura, homeopatia, medicina antroposofica e termalismo. Os serviços são oferecidos por iniciativa local, mas recebem financiamento do Ministério da Saúde (MS) por meio do Piso de Atenção Básica (AB) de cada município. Em 2016, mais de 2 milhões de atendimento utilizando PIC foram realizados em Unidades Básicas de Saúde (UBS), destes mais de 770 mil foram de Medicina Tradicional Chinesa (MTC), que inclui a acupuntura; 85 mil foram de fitoterapia e 13 mil de homeopatia e mais de 926 mil compreendem outras práticas (LOSSO LN, et al., 2017).

Após a introdução da PNPIC, o MS incentivou os estados e municípios a introduzirem essa política no âmbito do SUS. A partir publicação da Portaria no 1.654 de 2011, o MS com o intuito de melhorar a execução dos serviços da $A B$, utilizou-se o monitoramento e avaliação de processos e resultados, a contar do Programa 
Nacional de Melhoria do Acesso e da Qualidade da Atenção Básica (PMAQ-AB), que só foram iniciados no segundo ciclo, onde possibilitou-se à busca, compreensão e reconhecimento do empenho de equipes com experiências vitoriosas de implementação das PIC, estimulando a ampliação do acesso a essas práticas no pais, bem como o compromisso da gestão na implantação da política em questão (CARVALHO JL, et al., 2017).

No SUS, as PIC compreendem 29 procedimentos, que podem ser realizadas isoladas ou associada ao tratamento de inúmeras patologias, tais como estresse, ansiedade, cefaleia, fibromialgia, lombalgia, entre outras (MUNDIM BV, et al., 2020). Desde a sua implantação, a procura e o acesso dos usuários do SUS a essas práticas integrativas tem crescido exponencialmente (CARVALHO JL, et al., 2017).

Este movimento de crescimento deve-se a diversos fatores, entre eles o maior reconhecimento dessas práticas pelas evidências científicas e mesmo por sua efetividade pragmática facilmente verificável pelos benefícios; o crescente número de profissionais capacitados e habilitados; o reconhecimento e a valorização dos conhecimentos tradicionais de onde se originam grande parte destas práticas, sendo reconhecido inclusive pela OMS, que incentiva os as países a inserir estas práticas em seus sistemas de saúde, como feito no Brasil (GOTIJO MG, et al., 2017).

Ainda é desafiador o uso das PIC no SUS, uma vez que o Brasil ainda possui uma supervalorização e amplos incentivos à aplicação de insumos tecnológicos, sofisticados e de alto custo no cuidado ao paciente. Normalmente, os profissionais da saúde que possuem um conhecimento nas práticas clínicas e alternativas, estimulados pelo objetivo de consolidar uma concepção de cuidado em saúde, divergente do modelo biomédico padrão (foco na doença), encorajam-se em incentivar o uso das PIC e tornam-se profissionais importantes para a ampliação da implementação dessas práticas no SUS, onde, o ser humano é entendido em sua totalidade (LIMA CA, et al., 2018).

Considerando o contexto referido e a necessidade de avaliar a implantação na APS, através do PMAQ$A B$, o estudo propôs caracterizar as ofertas das Práticas Integrativas e Complementares (PIC) na Atenção Primária a Saúde no Estado do Pará.

\section{MÉTODOS}

O estudo desenvolvido foi do tipo transversal, avaliativo, com abordagem quantitativa, do tipo normativo, a partir do banco de dados de base nacional da etapa de avaliação externa do Programa de Melhoria do Acesso e da Qualidade da Atenção Básica (PMAQ-AB).

O PMAQ-AB é dividido por ciclos, onde: o Ciclo 1 (adesão), consiste na etapa formal de adesão ao programa, mediante contratualização de compromisso e indicadores firmados entre o MS e os gestores municipais; o Ciclo 2 (desenvolvimento), as equipes de saúde, gestores municipais e estaduais, além do MS, desenvolvem um conjunto de ações com o intuito de promover movimentos de mudança na gestão e no cuidado prestado pelas equipes, visando a melhoria do acesso e da qualidade da atenção a saúde; o Ciclo 3 (avaliação externa), consiste na avaliação externa das condições de acesso e de qualidade da totalidade de municípios e equipes participantes do programa.

O estudo foi constituído por 125 municípios do Estado do Pará, que aderiram e obtiveram registros de notificação na base de dados do PMAQ-AB, assim, apenas o segundo (2013 a 2014) e terceiro ciclo (2016 a 2018) do PMAQ-AB, possuíam dados procedentes do Estado do Pará, referente as Práticas Integrativas e Complementares. De acordo com dados do Instituto Brasileiro de Geografia e Estatística (2019), o Estado do Pará apresentava população estimada de 8.690 .745 habitantes, e com produto interno bruto (PIB) per capita de $R \$ 18.549,33$; possuindo uma área de 1.245.870,748 $\mathrm{Km}^{2}$

Foram incluídos o estudo as Unidades Básicas de Saúde (UBS), localizadas no Estado do Pará e que aderiram ao programa de avaliação proposto pelo MS e que responderam ao questionário do módulo IV. Foram excluídos os municípios que não estão localizados no Estado do Pará, que não aderiram ao programa de avaliação proposto pelo MS e que não responderam ao questionário do módulo IV. 
Os dados coletados foram do tipo quantitativo, gerados a partir do registro detalhado no preenchimento do formulário, e foram organizados por categoria para facilitar a interpretação dos resultados. Para a efetivação das análises foram realizados, primeiramente, momentos de leitura livre dos dados para um conhecimento geral dos resultados. Posteriormente, buscou-se identificar os temais mais significativos e recorrentes. A partir dessa identificação, procurou-se organizar os dados em categorias com vistas ao estabelecimento de conexão entre essas e os objetivos propostos pelo estudo, buscando aumentar a compreensão sobre o tema. A busca ocorreu através dos descritores presentes no banco de dados de base nacional da etapa de avaliação externa ao PMAQ-AB (Quadro 1).

Quadro 1 - Descritores utilizados na pesquisa.

\begin{tabular}{|c|c|c|}
\hline Componentes & Categoria & Subcategoria \\
\hline \multirow{2}{*}{ Organizacional } & Municípios & $\mathrm{Nc}$ \\
\cline { 2 - 3 } & Tipo de equipe & $\mathrm{Nc}$ \\
\hline \multirow{3}{*}{ Infra-estrutura } & Planta física & $\mathrm{Nc}$ \\
\cline { 2 - 3 } & Ambiência & $\mathrm{Nc}$ \\
\cline { 2 - 3 } & Condições estruturais & $\mathrm{Nc}$ \\
\hline \multirow{3}{*}{ Atividades } & Educação & Tipos de práticas \\
& Práticas Integrativas e & Ações para as práticas \\
\cline { 2 - 3 } & Complementares & $\mathrm{Nc}$ \\
\cline { 2 - 3 } & Apoio Organizacional & \\
\cline { 2 - 3 }
\end{tabular}

Legenda: Nc: nada consta no registro do PMAQ-AB. Fonte: Moreira MP, et al., 2021.

Os dados estão apresentados como média (desvio padrão), mediana e frequência de distribuição, pelo Software BioEstat 5.0, estabelecendo o nível de significância de 95\% ( $p>0.05)$.

\section{RESULTADOS}

Um total de 125 municípios paraenses foram incluídos no estudo, 39 relacionados ao Ciclo 2 e 86 pertencentes ao Ciclo 3, totalizando 86,8\% (125/144) de municípios existentes no Estado do Pará. As características basais dos municípios, de acordo com o seu perfil social, estrutura física e atividades estão apresentadas na (Tabela 1).

Tabela 1 - Características basais dos municípios paraenses de acordo com o ciclo de análise.

\begin{tabular}{|c|c|c|}
\hline Características & $\begin{array}{l}\%, \text { n (Ciclo 2) } \\
\text { (n = 39) }\end{array}$ & $\begin{array}{c}\%, n \text { (Ciclo 3) } \\
(n=86)\end{array}$ \\
\hline Total de municípios & $27,08(39)$ & $59,72(86)$ \\
\hline Todos aceitaram participar? & $100(39)$ & $100(86)$ \\
\hline Existe um responsável técnico? & $89,74(35)$ & $81,39(70)$ \\
\hline \multicolumn{3}{|l|}{ Estrutura física } \\
\hline Existem salas para reuniões? & $82,05(32)$ & $87,20(75)$ \\
\hline As salas são privativas? & $51,28(20)$ & $75,58(65)$ \\
\hline \multicolumn{3}{|l|}{ Educação Permanente } \\
\hline Realiza educação permanente para todos os profissionais? & $43,58(17)$ & $58,13(50)$ \\
\hline \multicolumn{3}{|l|}{ Atividades } \\
\hline $\begin{array}{c}\text { Realiza ações de Práticas Integrativas e /ou complementares e } \\
\text { assistência farmacêutica? }\end{array}$ & $89,74(35)$ & $93,02(80)$ \\
\hline $\begin{array}{c}\text { Ocorre o apoio para o desenvolvimento das Práticas Integrativas e } \\
\text { Complementares? }\end{array}$ & $17,94(7)$ & $43,02(37)$ \\
\hline
\end{tabular}

Fonte: Moreira MP, et al., 2021.

Conforme observa-se, dos 144 municípios existentes no Estado do Pará, apenas 125 obtiveram dados para serem incluídos no estudo, com prevalência significativa do Ciclo 3, que obteve 59,72\% (86/144) do total de municípios presentes. Todas as localidades aceitaram participar do estudo; apresentaram boa estrutura física e realizaram educação permanente com todos os profissionais que corroboraram com suas atividades. 
Em média, 91,38\% ( $\pm 2,31)$ dos municípios realizaram ações de PIC, porém, em sua totalidade, o apoio para o desenvolvimento dessas atividades foi baixo, tendo como média $30,48 \%$ ( $\pm 17,73)$, com um significativo aumento no Ciclo 3 - 43,02\% (37/86).

Com relação as atividades das PIC, a maior incidência do desenvolvimento das práticas integrativas, foram realizadas durante o Ciclo 2, totalizando apenas $12,81 \%$ do total de atividades. A medicina tradicional chinesa/acupuntura foi a que mais prevaleceu $(7,69 \%)$, tendo apenas três municípios que desenvolveram está atividade. No Ciclo 3, a porcentagem de práticas realizadas foi de 17,44\% e 15 municípios realizaram esta atividade, sendo à prática da medicina tradicional chinesa/auriculoterapia a única a ser realizada (Tabela 2).

Tabela 2 - Práticas Integrativas e Complementares realizadas nos 125 municípios analisados.

\begin{tabular}{ccc}
\hline PIC's & $\begin{array}{c}\text { \%, } \mathbf{n} \text { (Ciclo 2) } \\
\mathbf{( n = 3 9 )}\end{array}$ & $\begin{array}{c}\text { \%, } \mathbf{n} \text { (Ciclo 3) } \\
\text { (n=86) }\end{array}$ \\
\hline Medicina tradicional chinesa / acupuntura & $7,69(3)$ & $\mathrm{Nc}$ \\
\hline Medicina tradicional chinesa / auriculoterapia & $2,56(1)$ & $17,44(15)$ \\
\hline $\begin{array}{c}\text { Medicina tradicional chinesa / práticas corporais (Tai chi chuan, Lian } \\
\text { Gong, Chi Gong, Tui-Ná) e/ou mentais (meditação): }\end{array}$ & $0(0)$ & $\mathrm{Nc}$ \\
\hline Plantas medicinais e fitoterapia & $0(0$ & $\mathrm{Nc}$ \\
\hline Homeopatia & $0(0)$ & $\mathrm{Nc}$ \\
\hline Medicina antroposófica & $0(0)$ & $\mathrm{Nc}$ \\
\hline Termalismo social / crenoterapia & $2,56(1)$ & $\mathrm{Nc}$ \\
\hline Total & $\mathbf{1 2 , 8 1 ( 5 )}$ & $\mathbf{1 7 , 4 4 ( 1 5 )}$ \\
\hline
\end{tabular}

Legenda: Nc: nada consta no registro do PMAQ-AB. Fonte: Moreira MP, et al., 2021.

Após a publicação da Portaria do MS no 849/2017, que incluiu outras 14 práticas, houve um aumento significativo na porcentagem de práticas realizadas nos 125 municípios do Estado do Pará. O Ciclo 2, apresentou um índice de $23,05 \%$ e o Ciclo 3, de 116,2\% de avanço das atividades nos municípios paraenses (Tabela 3).

Tabela 3 - Desenvolvimento de outras Práticas Integrativas e Complementares realizadas nos 125 municípios analisados.

\begin{tabular}{ccc}
\hline PIC's & $\begin{array}{c}\text { \%, } \mathbf{n}(\mathbf{C i c l o ~ 2 )} \\
\mathbf{( n = 3 9 )}\end{array}$ & $\begin{array}{c}\text { \%, } \mathbf{n} \text { (Ciclo 3) } \\
\text { (n=86) }\end{array}$ \\
\hline Reiki & $2,56(1)$ & $0(0)$ \\
Yoga & $0(0)$ & $9,30(8)$ \\
17,Ayurveda & $0(0)$ & $1,16(1)$ \\
Florais & $0(0)$ & $3,48(3)$ \\
Sho-in/Shiatsu/Massoterapia/ Reflexologia & $2,56(1)$ & $11,62(10)$ \\
Shantala & $2,56(1)$ & $19,76(17)$ \\
Talassoterapia & $0(0)$ & $0(0)$ \\
Biodança & $0(0)$ & $5,81(5)$ \\
Musicoterapia & $0(0)$ & $17,44(15)$ \\
Dança circular & $5,12(2)$ & $17,44(15)$ \\
Naturologia & $0(0)$ & $0(0)$ \\
Terapia comunitária & $10,25(4)$ & $11,62(10)$ \\
Terapia com argila & $0(0)$ & $1,16(1)$ \\
Arterapia & $0(0)$ & $0(0)$ \\
Osteopatia & $0(0)$ & $5,81(5)$ \\
Quiropraxia & $\mathrm{Nc}$ & $8,13(7)$ \\
Total & $\mathrm{Nc}$ & $3,48(3)$ \\
\hline
\end{tabular}

Legenda: Nc: nada consta no registro do PMAQ-AB. Fonte: Moreira MP, et al., 2021. 
O desenvolvimento das PIC ocorre por diversos processos. No estudo, as consultas individualizadas dos profissionais no Núcleo Ampliado de Saúde da Família (NASF) demonstraram ser, as principais ações realizadas (Ciclo 2) nos municípios estudados, com 15,38\% das atividades; as consultas compartilhadas e reuniões em grupo terapêuticos, obtiveram o mesmo percentual $(10,25 \%)$, seguido de atendimentos domiciliares $(7,69 \%)$ e atividades de educação permanente $(5,12 \%)$.

O Ciclo 3, as atividades de educação permanente junto à equipe que compõe o NASF, apresentaram um crescimento de $59,99 \%$, comparado ao Ciclo 2 . As consultas individuais dos profissionais do NASF $(43,02 \%)$, reunião de grupos terapêuticos $(37,20 \%)$ e os atendimentos domiciliares $(25,58 \%)$, foram as atividades que mais despertaram interesse nos municípios estudados (Tabela 4).

Tabela 4 - Demonstrativo das principais ações que se dá o desenvolvimento das práticas integrativas e complementares nos municípios estudantes durante o Ciclo 2 e 3.

\begin{tabular}{ccc}
\hline Ações & $\begin{array}{c}\text { \%, n (Ciclo 2) } \\
\text { (n= 39) }\end{array}$ & $\begin{array}{c}\text { \%, n (Ciclo 3) } \\
\text { (n=86) }\end{array}$ \\
\hline Consultas individuais dos profissionais do NASF & $15,38(6)$ & $43,02(37)$ \\
Consultas compartilhadas & $10,25(4)$ & $13,95(12)$ \\
Atendimento domiciliares & $7,69(3)$ & $25,58(22)$ \\
Grupos terapêuticos & $10,25(4)$ & $37,20(32)$ \\
Atividades de educação permanente nestes temas para a equipe & $5,12(2)$ & $65,11(56)$ \\
\hline
\end{tabular}

Fonte: Moreira MP, et al., 2021.

\section{DISCUSSÃO}

O Sistema Único de Saúde (SUS), criado em 1990, contribuiu para a reestruturação da assistência à saúde no Brasil, com o acesso amplo aos serviços, diminuição de doenças, melhores condições sanitárias e aumentando a expectativa de vida dos brasileiros (SOUSA IM, et al., 2016).

As PIC, utilizam terapias simples e menos dependentes de tecnologias, mas, com igual ou superior eficácia nas situações comuns de adoecimento. Esta terapia, restitui o paciente como o centro do cuidado, favorecendo o vínculo terapêutico e proporcionando autocuidado (LIMA CA, et al., 2018).

As características basais do estudo, corroboram com perfil epidemiológico em outros municípios brasileiros, onde destaca-se as transformações ocorridas nas últimas décadas no perfil de assistência no SUS, onde não mais somente o medicamento como insumo essencial, mas a sua relação com o usuário, na busca da efetivação da assistência terapêutica integral (MANTOVANI MF, et al., 2016). Porém, apesar da região norte possuir inúmeras riquezas naturais, sociais e culturais, é provável que muitas práticas integrativas sejam realizadas por raizeiros, curandeiros, benzedeiros e xamãs, à margem do sistema público de saúde e consequentemente, sem o aparecimento de casos notificados (BARBOSA FE, et al., 2020).

Nesse estudo, observou-se que 85,56\% $( \pm 5,90)$ das Unidades Básicas de Saúde (UBS) avaliadas nos municípios paraenses possuíam responsáveis técnicos, demonstrando um importante avanço no ponto de partida para uma assistência à saúde qualificada. Em estudos realizados por Ruela LO, et al. (2019) com dados do Ciclo 1 do PMAQ-AB, evidenciou-se que apenas 20,1\% das UBS no Brasil que realizavam serviços clínicos, possuíam responsáveis técnicos no serviço, como parte integrante da equipe.

Ao comparar-se com os dados do Ciclo 2 e 3, é possível perceber um considerável aumento destes profissionais tão importantes para a operacionalização da assistência, porém, ainda, estamos muito distantes do ideal. A medida em que se pensa na assistência à saúde qualificada, cada vez mais trazem o profissional como fundamental no processo assistencial (TESSER CD, et al., 2018).

Pelas informações prestadas, verificou-se que, no Ciclo 2, 82,05\% dos municípios possuíam salas para os profissionais de saúde se reunirem e planejarem suas ações, porém apenas $51,28 \%$ eram salas privativas. Consideravelmente, houve um grande avanço no Ciclo 3. Quanto a estrutura física, o Ciclo 2 e Ciclo 3, apresentaram $87,2 \%$ e $75,58 \%$, respectivamente, comparando-se, aos mesmos quesitos visualizados no 
Ciclo 2. A estruturas apropriada nos serviços de saúde gera condições básicas para obter-se um bom desempenho na assistência prestada aos usuários. Portanto, faz-se necessário ampliar a investigação sobre as condições da organização e estrutura das unidades básicas de saúde para atender os pacientes e seus usuários do sistema público de saúde (CARVALHO JL e NÓBREGA MP, 2017).

Conforme observado no estudo, a prática de educação permanente ocorre, porém, ainda é escasso frente aos municípios. Os estudos que contribuem nessa direção apontam a fragilidade do impacto das capacitações na qualidade dos serviços de saúde. Assim, destaca-se a implantação de Educação Permanente em Saúde (EPS) como política nacional para formação e desenvolvimento de trabalhadores da saúde, tendo em vista a articulação entre as possibilidades de desenvolver a educação dos profissionais e a ampliação da capacidade resolutiva dos serviços (GARNELO L, et al., 2018).

Nos últimos anos, a população mundial atraiu-se pela utilização de Práticas Não-Convencionais em Saúde (PNCS), incentivando as entidades à implementação e ao desenvolvimento de medidas que visem a corresponder aos anseios da sociedade nessa área (SOUSA IM e TESSER CD, 2017).

Diferenciando do modelo biomédico, as PIC são vistas como um importante método de assistência à saúde, por considerar o indivíduo em sua integralidade. Na maioria, a procura por esta prática se dá por diversos fatores, tais como: baixos efeitos adversos, complemento na terapia alopática, acolhimento e escuta qualificada, valores, crenças e outros. Além disso, as PIC possuem um importante potencial para redução do consumo de medicamentos (MORÉ ao, et al., 2016).

No estudo, foi observado uma baixa adesão no uso das PIC nos municípios. Houve, o predomínio da medicina tradicional chinesa (7,69\% - Ciclo 2; 17,44\% - Ciclo 3); Terapias Comunitárias (10,25\% - Ciclo 2; 11,62\% - Ciclo 3); Do-in/Shiatsu/ Massoterapia (11,62\% - Ciclo 3); Shantala (19,76\% - Ciclo 3); Musicoterapia e Dança Circular (17,44\% - Ciclo 3). Sabemos que ainda estamos longe de atingir o ideal. Mesmo com todos os avanços, esta prática ainda precisa de muitos incentivos para ser praticada e aderida junto a população e ao sistema.

Em alguns estudos, a utilização da fitoterapia e da homeopatia aparecem em ascensão. A acupuntura foi investigada de modo isolado apenas por Oliveira VB, et al. (2018). Entretanto, tanto a homeopatia quanto a acupuntura, se destacam como aquelas que apresentaram maior adesão pelos usuários e maior oferta pelos serviços (RANGEL CT, et al., 2016; SANTOS MS, et al., 2018). Esse fato vai ao desencontro de dados apresentados em nosso estudo, onde apenas à prática da acupuntura foi demonstrado nos municípios catalogados.

Uma vez que a atenção primária à saúde é a porta de entrada do usuário para a rede de atenção ao cuidado em saúde, de acordo com a Política Nacional de Atenção Básica (PNAB), é possível concluir que esse nível de atenção constitui, locus privilegiado para a implementação das PIC no sistema público de saúde brasileiro. De fato, dados do MS apontam que as PIC são ofertadas, em sua grande maioria, nos serviços de atenção básica (GOTIJO MG, et al., 2017).

Foi demonstrado, em um estudo realizado na cidade de Florianópolis, Santa Catarina - Brasil, que os profissionais da atenção primária incentivam o uso das PIC durante a consulta com o paciente e normalmente, iniciam o tratamento logo que possível (CARVALHO JL, et al., 2017).

No SUS, o uso das PIC necessita de recursos humanos capacitados para o serviço. Nesse contexto, a qualificação profissional é fundamental para o processo de implementação das práticas. O desconhecimento da PNPIC, bem como das terapias abordadas na política dificulta a adesão, tanto de profissionais quanto dos serviços, na oferta das práticas (TESSER CD, et al., 2018).

No Brasil, os profissionais da saúde, como médicos, enfermeiros, fisioterapeutas, farmacêuticos, entre outros, são habilitados para a prática. Porém, a baixa adesão em capacitações e a deficiência no ensino durante a formação, dificultam o melhor aperfeiçoamento dos profissionais da saúde, embora muitos demonstrem interesse na capacitação e concordância com o uso das práticas nos serviços (THIAGO SC e TESSER CD, 2011; LARA AC, et al., 2019). 
A expansão das práticas integrativas no SUS, foi mais expressivo a partir do desenvolvimento das atividades por profissionais não médicos, o que exigem dos outros profissionais uma ampliação dos conhecimentos sobre as PIC. Portanto, há uma necessidade de incentivo de modo a resgatar a dimensão humanística do atendimento a saúde. Esse fato pode justificar o aumento do desenvolvimento das práticas no Ciclo 3 (RUELO LO, et al., 2019).

Todas as ações desenvolvidas para o desenvolvimento das PIC, foram atribuídas a vários fatores, dentre elas, consultas individuais, compartilhadas; atendimento domiciliares, reunião de grupos terapêutica e atividades de educação permanente. Apesar do aumento da inserção das práticas nos últimos anos, o seu potencial terapêutico e suas contribuições para saúde ainda são pouco explorados no SUS. Mesmo que o MS tenha avaliado de modo positivo esse aumento, existem lacunas, como a avaliação das PIC nos serviços e melhor acompanhamento do impacto causado pela política (MATTOS G, et al., 2018).

Além disso, a preeminência do modelo biomédico atual somado à tendência mercadológica na área da saúde, que transforma os saberes e prática em mercadorias, pode ser uma importante limitação nos avanços esperados para essa prática. Desse modo, existe o desafio de aprofundar o cuidado em um modelo integral de assistência, superando a supremacia da lógica de serviços baseados na biomedicina (SOUSA IV, et al., 2019).

\section{CONCLUSÃO}

A partir do estudo, podemos concluir que o Estado do Pará apresenta uma crescente na oferta das PIC na Atenção Primária à Saúde e a publicação da Portaria do MS no 849/2017, favoreceu esse aumento em todos os municípios estudados. Por mais que os profissionais possuam interesse na realização, investimentos na área necessitam ser realizados, de modo que ocorra uma participação ativa dos gestores, profissionais da saúde, pacientes e instituições educacionais.

\section{REFERÊNCIAS}

1. BARBOSA FE, et al. Oferta de Práticas Integrativas e Complementares em Saúde na Estratégia Saúde da Família no Brasil, 2020; 36: e00208818.

2. BRASIL. Ministério da Saúde (MS). Manual de implantação de serviços de práticas integrativas e complementares no SUS. Brasília: MS; 2018.

3. BRASIL. Ministério da Saúde. Portaria no 145, de 11 de janeiro de 2017, Brasília, 2017.

4. CARVALHO JL, NÓBREGA MP. Práticas integrativas e complementares como recurso de saúde mental na Atenção Básica, 2017; 38: e2017-0014.

5. CARVALHO JLS e NÓBREGA MPSS. Práticas integrativas e complementares como recurso de saúde mental na Atenção Básica. Revista Gaúcha de Enfermagem, 2017; 4: 1-9.

6. GARNELO L, et al. Acesso e cobertura da atenção primária à saúde para populações rurais e urbanas na região Norte do Brasil. Saúde Debate, 2018; 42: 81-99.

7. GONTIJO MBA, NUNES MF. Práticas Integrativas E Complementares: Conhecimento e Credibilidade de Profissionais do Serviço Público de Saúde. Revista Trabalho, Educação e Saúde, 2017; 15: 301-320.

8. LARA AC, et al. Avaliação do consumo de produtos naturais por usuários de estratégias de saúde da família do município de Rondonópolis - MT. Revista UniVap, 2019; 25: 98-109.

9. LIMA CA, et al. Integrative and complementary practices: use by Community health agents in self-care. Revista Brasileira de Enfermagem, 2018; 71 (Supl. 6): 2682-2688.

10. LOSSO LN e FREITAS SFT. Avaliação do grau da implantação das práticas integrativas e complementares na Atenção Básica em Santa Catarina, Brasil. Revista Saúde em Debate, 2017; 41: 171-187.

11. MANTOVANI MF, et al. Utilização de Terapias Complementares por pessoas com Hipertensão Arterial Sistêmica. Revista Baiana de Enfermagem, 2016; 30: 1-8.

12. MATTOS G, et al. Plantas medicinais e fitoterápicos na Atenção Primária em Saúde: percepção dos profissionais. Ciência \& Saúde Coletiva, 2018; 23:3735-3744.

13. MORÉ $A O$, et al. Integrating acupuncture into primary health care: the experience of an education model implemented within the Brazilian Unified Health System in Florianópolis. Acupuncture in Medicine, 2016; 34: 476-481.

14. MUNDIM, BV, et al. Práticas integrativas e complementares realizadas em pacientes com lombalgia em uma Unidade Básica de Saúde na região noroeste do Paraná: relato de experiência. Revista Eletrônica Acervo Saúde, 2020; 43: e2470.

15. OLIVEIRA VB, et al. Conhecimento e Uso de Plantas Medicinais por Usuários de Unidades Básicas de Saúde na Região de Colombo, PR. Revista Brasileira de Ciências da Saúde, 2018; 22: 57-64. 
16. RANGEL CT, et al. A terapia comunitária integrativa e a enfermagem: o fenômeno e seus contextos. Revista de Pesquisa Cuidado é Fundamental Online, 2016; 8: 3770-3779.

17. RUELA LO, et al. Implementação, acesso e uso das práticas integrativas e complementares no Sistema Único de Saúde: revisão da literatura. Ciência \& Saúde Coletiva, 2019; 24: 4239-4250.

18. SANTOS MS, et al. Práticas Integrativas e Complementares: avanços e desafios para a promoção da saúde de idosos. Revista Mineira de Enfermagem, 2018; 22: 1-5.

19. SOUSA IM, TESSER CD. Medicina Tradicional e Complementar no Brasil: inserção no Sistema Único de Saúde e integração com a atenção primária. Caderno de Saúde Pública, 2017; 33: e00150215.

20. SOUSA IM, TESSER CD. Medicina Tradicional e Complementar no Brasil: inserção no Sistema Único de Saúde e integração com a atenção primária. Caderno de Saúde Pública, 2016; 33: 1-15.

21. SOUSA IV, et al. Coping with problems that impact on the health hof a socially vulnerable community from the resident's perspective. Ciência \& Saude Colet, 2019; 24: 1647-1656.

22. SOUSA LA, BARROS NF. Integrative and Complementary Practices in the Unified Health System: progresses and challenges. Revista Latino Americana de Enfermagem, 2018; 26:e3041.

23. SOUZA IN, et al. Produção científica acerca da Política Nacional de Práticas Integrativas e Complementares. Revista Eletrônica Acervo Saúde, 2020; 12: e4386.

24. TESSER CD, et al. Práticas Integrativas e Complementares na Atenção Primária à Saúde brasileira. Saúde em Debate., 2018; 42: 174-188.

25. THIAGO SC, TESSER CD. Percepção de médicos e enfermeiros da Estratégia de Saúde da Família sobre terapias complementares. Revista de Saúde Publica, 2011; 45:249- 257. 